\title{
Les réformes de la NDRC, ou les mues de la planification centrale
}

\section{Thibaud Voïta}

\section{(2) OpenEdition \\ Journals}

Édition électronique

URL : http://journals.openedition.org/perspectiveschinoises/4163

ISSN : 1996-4609

\section{Éditeur}

Centre d'étude français sur la Chine contemporaine

\section{Édition imprimée}

Date de publication : 1 avril 2008

Pagination : 112-113

ISSN : 1021-9013

Référence électronique

Thibaud Voïta, «Les réformes de la NDRC, ou les mues de la planification centrale », Perspectives chinoises [En ligne], 2008/2 | 2008, mis en ligne le 01 avril 2011, consulté le 06 mai 2019. URL : http:// journals.openedition.org/perspectiveschinoises/4163 


\title{
Les réformes de la NDRC, ou les mues de la planification centrale
}

\author{
Analyse de Thibaud Voïta à partir de : \\ - Luo Changpin et al., "Une silhouette commence à apparaître ", Caijing, 17 mars 2008. \\ - Zuo Qinglin, " Le Retour de la NDRC à ses fonctions premières ", 21 Shiji jingji baodao, 18 mars 2008. \\ - Wang Dongming, "Une nouvelle étape dans la réforme de la gestion administrative et des institutions gouvernementales ", \\ Qiu shi, ler avril 2008.
}

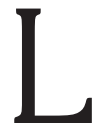
a Commission nationale pour le développement et la réforme (National Development and Reform Commission, NDRC) est surnommée le " ministère des ministères " (buzhong zhi bu) ou encore le " premier grand ministère chinois » (Zhongguo diyi «dabu »). D'après Caijing, la NDRC a été, depuis sa création, au centre des réformes. Elle a émergé en 1988, sous le nom de Commission de la planification nationale à la suite de la fusion de la Commission de la planification nationale avec la Commission de l'économie nationale Entre 1993 et 1998, elle a joué un rôle de régulation macroéconomique entre les différents ministères avant de devenir si puissante, à la fin des années 1990, qu'elle fut surnommée le " petit Conseil des Affaires d'État " (xiao guowuyuan).

La NDRC, sous son nom actuel, a été créée en 2003, avec des compétences élargies. Elle s'est retrouvée en charge de dossiers aussi importants que la régulation macroéconomique, les réformes administratives, l'allocation des ressources, le contrôle des prix... Ses fonctions s'étendaient jusqu'à fixer les jours des vacances nationales. Au moins $28 \mathrm{mi}-$ nistères et institutions du Conseil des Affaires d'État ont des échanges quotidiens avec la NDRC.

Les réformes ayant touché la commission lors de la dernière session de l'Assemblée nationale du peuple (ANP) remettent en question bon nombre de ses attributs. Officiellement, il s'agit d'un recentrage : la NDRC se trouve privée de ses activités microéconomiques et d'approbation des projets pour se concentrer sur les politiques macroéconomiques. "Notre travail va être plus précis ", affirment par euphémisme les cadres de la NDRC.

La carrière du nouveau ministre promu à la tête de la NDRC, Zhang Ping, est révélatrice de l'orientation des réformes. Né dans le nord de l'Anhui en 1946, Zhang Ping a été confronté aux réalités de la misère paysanne. Il a effectué toute sa carrière dans sa province natale avant de monter à Pékin, et s'est fortement engagé dans la résolution des problèmes ruraux. On retient principalement de lui ses réformes fiscales; dans sa province, on parle de lui comme de "la première personne à s'être souciée de la fiscalité rurale "

Il a rejoint la NDRC en 2005, en tant que ministre adjoint en charge de la gestion de l'économie, du commerce et des prix. En tant que tel, il était à la tête des directions du commerce, de l'inspection des prix et de la réforme du système de gestion harmonieuse de l'économie, ainsi que du Centre de certification des prix, du Centre d'examen des prix, des Éditions du marché chinois, du Centre d'évaluation des prix des médicaments, et enfin du Bureau national des céréales. Il s'est principalement illustré dans ces fonctions par le contrôle des frais liés à l'éducation, par la baisse des prix des soins médicaux, le contrôle des prix de l'immobilier, la réforme des prix de l'énergie, la stabilisation des prix agricoles. Il semblerait que ce sont les départements à la tête desquels il se trouvait qui vont désormais constituer le cœur de la NDRC, les autres ayant été pour la plupart détachés de la commission.

Caijing se félicite de ces réformes. La NDRC était une trop grosse structure, la nouvelle répartition des pouvoirs devrait limiter ses compétences en matière d'autorisations d'investissements en les redistribuant à des administrations locales. La répartition des pouvoirs entre localités et centre devrait s'en trouver clarifiée ; les gouvernements locaux vont être responsabilisés. En outre, la redistribution de certaines de ces compétences à de grands ministères est également considérée comme un point positif. Caijing insiste sur cette nouvelle répartition de compétences dans le secteur de l'énergie, qui concerne principalement quatre institutions :

1. Un Energy Leading Group, dirigé par Wen Jiabao.

2. Le bureau de ce Leading Group, au sein même de la NDRC, qui devra traiter les affaires courantes.

3. Le Bureau de l'énergie de la NDRC. 
4. La Commission de régulation de l'électricité, créée en 2003.

Désormais, le Bureau de l'énergie sera hiérarchiquement à un rang plus élevé que la NDRC. Zhang Guobao, ancien vice-ministre de la NDRC chargé de l'énergie, a bon espoir d'en devenir le responsable.

Quelles conclusions tirer de cette réorganisation? En premier lieu, Wang Dongming insiste sur la transition vers un gouvernement de services (fuwuxing zhengfu) perçu comme un étape essentielle pour construire, à l'horizon 2020, la "société socialiste de type chinois". On remarquera que, durant sa carrière, Zhang Ping s'est constamment soucié des questions rurales. Il était également en charge des différents centres de gestion des prix lorsqu'ont été prises, il y a quelques mois, les mesures de contrôle des prix. La planification centrale semble donc s'orienter vers une meilleure gestion des prestations sociales des pouvoirs publics, pour mieux partager les fruits de la croissance. En second lieu, la réduction des prérogatives de la NDRC ne signifie pas obligatoirement moins d'interventionnisme dans l'économie de la part du pouvoir central. Au contraire, ce recentrage doit permettre à la NDRC de se consacrer plus pleinement aux contrôles macroéconomiques. Au lendemain de cette session de l'ANP, le mot d'ordre est donc à la rationalisation de l'action de l'État. •

\section{Les principales réformes de l'administration chinoise depuis l'ouverture du pays. Source : d'après Caijing.}

1982 : allègement de l'appareil administratif ; le nombre d'institutions sous contrôle du Conseil des Affaires d'État passe de 100 à 61 .

1988 : nouvelle diminution du nombre des institutions ; réduction du personnel administratif de $19,2 \%$.

1993 : objectif de l'édification du "socialisme de marché "; mise en place de la séparation entre le gouvernement et les entreprises. Les institutions directement placées sous le contrôle du Conseil des Affaires d'État passent de 86 à 59 , soit une diminution de $20 \%$.

1998 : transition des fonctions du gouvernement, lancement de la réforme des entreprises. Le nombre d'institutions dépendant du Conseil des Affaires d'État passe de 40 à 29, certaines fonctions sont transférées aux entreprises, aux gouvernements locaux, à la population. Le personnel diminue de moitié. Création de la Commission of Science, Technology, and Industry for National Defense (Costind), des ministères de l'Information, de la Protection sociale et des Travailleurs, de la Propriété de la terre.

2003 : les responsabilités du gouvernement sont désormais de réguler l'économie, le marché, la société et les affaires publiques. Mise en place de la Sasac (State Owned Assets Supervision and Administration Commission), de la NDRC, de la China Banking Regulatory Commission (CBRC) ; réorganisation du ministère du Commerce, de la State Food and Drug Administration. La State Administration of Work Safety est placée sous le contrôle direct du Conseil des Affaires d'État.

\section{Caractères chinois}

buzhong zhi bu 部中之部

Zhongguo diyi «dabu »中國第一《大部》

xiao guowuyuan 小國務院

fuwuxing zhengfu 服務型政府 